\title{
Fermions Tunneling from Higher-Dimensional Reissner-Nordström Black Hole: Semiclassical and Beyond Semiclassical Approximation
}

\author{
ShuZheng Yang, ${ }^{1}$ Dan Wen, ${ }^{1,2,3}$ and Kai Lin ${ }^{1,4}$ \\ ${ }^{1}$ Department of Astronomy, China West Normal University, Nanchong, Sichuan 637002, China \\ ${ }^{2}$ College of Physics and Information Science, Hunan Normal University, Changsha, Hunan 410081, China \\ ${ }^{3}$ Faculdade de Engenharia de Guaratinguetá, Universidade Estadual Paulista, 12516-410 Guaratinguetá, SP, Brazil \\ ${ }^{4}$ Instituto de Física e Química, Universidade Federal de Itajubá, 37500-903 Itajubá, MG, Brazil
}

Correspondence should be addressed to Kai Lin; lk314159@hotmail.com

Received 20 April 2016; Accepted 25 July 2016

Academic Editor: Mir Faizal

Copyright (c) 2016 ShuZheng Yang et al. This is an open access article distributed under the Creative Commons Attribution License, which permits unrestricted use, distribution, and reproduction in any medium, provided the original work is properly cited. The publication of this article was funded by SCOAP ${ }^{3}$.

Based on semiclassical tunneling method, we focus on charged fermions tunneling from higher-dimensional Reissner-Nordström black hole. We first simplify the Dirac equation by semiclassical approximation, and then a semiclassical Hamilton-Jacobi equation is obtained. Using the Hamilton-Jacobi equation, we study the Hawking temperature and fermions tunneling rate at the event horizon of the higher-dimensional Reissner-Nordström black hole space-time. Finally, the correct entropy is calculation by the method beyond semiclassical approximation.

Hawking radiation is an important prediction in modern gravitation theory [1-5]. Recently, Kraus et al. proposed quantum tunneling theory to explain and study Hawking radiation [6-31], and then semiclassical Hamilton-Jacobi method is put forward to research the properties of scalar particles' tunnels [32-36]. In 2007, Kerner and Mann investigated the $1 / 2$ spin fermion tunneling from static black holes [37]. In their work, the spin up and spin down cases are researched, respectively, and the radial equations are obtained, so that they can finally determine the Hawking temperature and tunneling rate at the event horizon. Subsequently, Kerr and Kerr-Newman black holes cases, the charged dilatonic black hole case, the de Sitter horizon case, the BTZ black hole case, 5-dimensional space-time cases, and several nonstationary black hole cases were all researched, respectively [38-48], and we used Hamilton-Jacobi method to study the fermion tunneling from higher-dimensional uncharged black holes $[49,50]$. However, up to now, no one has studied higher-dimensional charged black holes cases, so we set out to research that case. In our work, we developed the Kerner and Mann method and proved that the semiclassical Hamilton-Jacobi equation can be obtained not only with the Klein-Gordon equation of curved space-time, but also with the Dirac equation in curved space-time. Applying the Hamilton-Jacobi equation, we can then obtain semiclassical Hawking temperature and tunneling rate at the event horizon of higher-dimensional Reissner-Nordström black hole.

In modern physics theory, the concept of an extra dimension can help to solve some theoretical issues, so several higher-dimensional metrics of curved space-time were investigated. The metric of static charged $(n+2)$-dimensional Reissner-Nordström black hole is given by [10, 51-55]

$$
d s^{2}=-f(r) d t^{2}+f^{-1}(r) d r^{2}+r^{2} d \Omega_{n}^{2},
$$

where $d \Omega_{n}^{2}$ is the metric of $n$-dimensional sphere

$$
\begin{aligned}
d \Omega_{n}^{2}= & \sum_{i=1}^{n} h^{i i} d \theta_{i}^{2} \\
= & d \theta_{1}^{2}+\sin ^{2} \theta_{1} d \theta_{2}^{2}+\sin ^{2} \theta_{1} \sin ^{2} \theta_{2} d \theta_{3}^{2}+\cdots \\
& +\prod_{i=1}^{n-1} \sin ^{2} \theta_{i} d \theta_{n}^{2}
\end{aligned}
$$




$$
f(r)=1-\frac{\omega_{n} M}{r^{n-1}}+\frac{\omega_{n} Q^{2}}{2(n-1) V_{n} r^{2 n-2}}, \quad \omega_{n}=\frac{16 \pi}{n V_{n}},
$$

where $M$ and $Q$ are mass and electric charge of black hole, and the electromagnetic potential is

$$
A_{\mu}=\left(\frac{Q}{(n-1) V_{n} r^{n-1}}, 0,0,0, \ldots\right),
$$

where $V_{n}$ is volume of unit $n$-sphere (we can adopt the units $G=c=\hbar=1)$. The outer/inner horizon located at

$$
r_{ \pm}^{n-2}=\frac{\omega_{n}}{2}\left[M \pm \sqrt{M^{2}-\frac{n Q^{2}}{8 \pi(n-1)}}\right] .
$$

Obviously, at the horizons, the equation $f\left(r_{ \pm}\right)=0$ should be satisfied. However, the physical property near the inner horizon cannot be researched, so we just study the fermion tunneling at the outer event horizon of this black hole. The charged Dirac equation in curved space-time is

$$
\gamma^{\mu} D_{\mu} \Psi+\frac{m}{\hbar} \Psi=0, \quad \mu=t, r, \theta_{1}, \ldots, \theta_{n},
$$

where

$$
\begin{aligned}
& D_{\mu}=\partial_{\mu}+\Gamma_{\mu}+\frac{i q A_{\mu}}{\hbar} \\
& \Gamma_{\mu}=\frac{1}{8}\left[\widetilde{\gamma}^{a}, \widetilde{\gamma}^{b}\right] e_{a}^{v} e_{b v ; \mu},
\end{aligned}
$$

where $m$ and $q$ are mass and electric charge of the particles, and $e_{b v ; \mu}=\partial_{\mu} e_{b v}-\Gamma_{\mu \nu}^{\alpha} e_{a b}$ is the covariant derivative of tetrad $e_{b v}$. The gamma matrices in curved space-time need to be satisfied

$$
\left\{\gamma^{\mu}, \gamma^{\nu}\right\}=2 g^{\mu \nu} I
$$

After the gamma matrices are defined, we choose the gamma matrices in $(n+2)$-dimensional flat space-time as

$$
\begin{aligned}
& \tilde{\gamma}_{m \times m}^{1}=\left(\begin{array}{cc}
I_{m / 2 \times m / 2} & 0 \\
0 & -I_{m / 2 \times m / 2}
\end{array}\right) \\
& \widetilde{\gamma}_{m \times m}^{2}=\left(\begin{array}{cc}
0 & I_{m / 2 \times m / 2} \\
I_{m / 2 \times m / 2} & 0
\end{array}\right) \\
& \tilde{\gamma}_{m \times m}^{\eta}=\left(\begin{array}{cc}
0 & i \widetilde{\gamma}_{m / 2 \times m / 2}^{\eta-2} \\
-i \widetilde{\gamma}_{m / 2 \times m / 2}^{\eta-2} & 0
\end{array}\right), \\
& \quad \eta=3,4,5, \ldots, n+2,
\end{aligned}
$$

where $I_{m / 2 \times m / 2}$ and $\widetilde{\gamma}_{m / 2 \times m / 2}^{\eta}$ are unit matrices and flat gamma matrices with $m / 2 \times m / 2$ order and $m=2^{(n+2) / 2}$ is the order of the matrices in even (odd) dimensional space-time.
Corresponding to the flat case, the gamma matrices can be chosen as

$$
\begin{aligned}
\gamma_{m \times m}^{t} & =\frac{i}{\sqrt{f}} \widetilde{\gamma}_{m \times m}^{1} \\
\gamma_{m \times m}^{r} & =\sqrt{f} \widetilde{\gamma}_{m \times m}^{2} \\
\gamma_{m \times m}^{\eta} & =r^{-1} \sqrt{h^{\eta \eta}} \widetilde{\gamma}_{m \times m}^{\eta}, \quad \eta=3,4,5, \ldots, n+2 .
\end{aligned}
$$

Now, let us simplify the Dirac equation via semiclassical approximation, and rewrite the spinor function as

$$
\Psi=\left(\begin{array}{c}
A_{m / 2 \times 1}\left(t, r, \ldots, x^{\eta}, \ldots\right) \\
B_{m / 2 \times 1}\left(t, r, \ldots, x^{\eta}, \ldots\right)
\end{array}\right) e^{(i / \hbar) S\left(t, r, \ldots, x^{\eta}, \ldots\right)},
$$

where $A_{m / 2 \times 1}\left(t, r, \ldots, x^{\eta}, \ldots\right)$ and $B_{m / 2 \times 1}\left(t, r, \ldots, x^{\eta}, \ldots\right)$ are column matrices with $m / 2 \times 1$ order and $S$ is classical action. Via semiclassical approximation method, Substituting (10) into (5) and dividing the exponential term and multiplying by $\hbar$, we can get

$$
\left(\begin{array}{cc}
C & D \\
E & F
\end{array}\right)\left(\begin{array}{c}
A_{m / 2 \times 1} \\
B_{m / 2 \times 1}
\end{array}\right)=0
$$

$$
\begin{aligned}
C= & -\frac{1}{\sqrt{f}}\left(\frac{\partial S}{\partial t}+q A_{t}\right) I_{m / 2 \times m / 2} \\
& +m I_{m / 2 \times m / 2} \\
D= & i \sqrt{f} \frac{\partial S}{\partial r} I_{m / 2 \times m / 2} \\
& -\sum_{\eta} r^{-1} \sqrt{h^{\eta \eta}} \frac{\partial S}{\partial x^{\eta}} \widetilde{\gamma}_{m / 2 \times m / 2}^{\eta-2} \\
E= & i \sqrt{f} \frac{\partial S}{\partial r} I_{m / 2 \times m / 2} \\
& +\sum_{\eta} r^{-1} \sqrt{h^{\eta \eta}} \frac{\partial S}{\partial x^{\eta}} \widetilde{\gamma}_{m / 2 \times m / 2}^{\eta-2} \\
F= & \frac{1}{\sqrt{f}}\left(\frac{\partial S}{\partial t}+q A_{t}\right) I_{m / 2 \times m / 2} \\
& +m I_{m / 2 \times m / 2} .
\end{aligned}
$$

Solving (11), we have

$$
\begin{aligned}
& \left(E-F D^{-1} C\right) A_{m / 2 \times 1}=0 \\
& \left(F-E C^{-1} D\right) B_{m / 2 \times 1}=0 .
\end{aligned}
$$

It is evident that the coefficient matrices of (16) must vanish, when $A_{m / 2 \times 1}$ and $B_{m / 2 \times 1}$ have nontrivial solutions. Due to the fact that $C D=D C$, we can write the condition that determinant of coefficient vanish as

$$
\operatorname{det}(E D-F C)=0 \text {. }
$$


From the relation of flat gamma matrices $\left\{\widetilde{\gamma}^{\mu}, \tilde{\gamma}^{\nu}\right\}=2 \delta_{\mu \nu}$, we can obtain the semiclassical Hamilton-Jacobi equation in $(n+$ 2)-dimensional Reissner-Nordström space-time

$$
\begin{aligned}
& -\frac{1}{f}\left(\frac{\partial S}{\partial t}+q A_{t}\right)^{2}+f\left(\frac{\partial S}{\partial r}\right)^{2}+\cdots+g^{\eta \eta}\left(\frac{\partial S}{\partial x^{\eta}}\right) \\
& +\cdots+m^{2}=0
\end{aligned}
$$

Using the Hamilton-Jacobi equation, in charged static spacetime, we can separate the variables for the action as

$$
S=-\omega t+R(r)+Y\left(\ldots, x^{\eta}, \ldots\right)+K,
$$

( $K$ is a constant)

and the Hamilton-Jacobi equation is broken up as

$$
\begin{aligned}
-\frac{1}{f}\left(\omega-q A_{t}\right)^{2}+f\left(\frac{d R}{d r}\right)^{2}+m^{2} & =\frac{\lambda}{r^{2}} \\
\sum_{\eta} h^{\eta \eta}\left(\frac{\partial Y}{\partial x^{\eta}}\right)^{2}+\lambda & =0,
\end{aligned}
$$

where (20) and (21) are radial and nonradial equations, respectively, and $\lambda$ is a constant. However, we only research on the radial equation, because the tunnel at the event horizon is radial. From (20), we can get

$$
\frac{d R(r)}{d r}= \pm \frac{\sqrt{\left(\omega-q A_{t}\right)^{2} r^{2}+f\left(\lambda-m^{2} r^{2}\right)}}{f r}
$$

Near the event horizon, the radial action is given by

$$
\operatorname{Im} R_{ \pm}= \pm \frac{\pi\left(\omega-\omega_{0}\right)}{f^{\prime}\left(r_{+}\right)}+\operatorname{Im} C
$$

where $R_{+}$is part of the outgoing solution, while $R_{-}$is the part of incoming solution, and

$$
\omega_{0}=q \frac{Q}{(n-1) V_{n} r_{+}^{n-1}} .
$$

So the tunneling rate is

$$
\begin{aligned}
\Gamma & =\frac{\operatorname{Prob}[\text { out }]}{\operatorname{Prob}[\text { in }]}=\frac{\exp \left(-2 \operatorname{Im} S_{+}\right)}{\exp \left(-2 \operatorname{Im} S_{-}\right)} \\
& =\frac{\exp \left(-2 \operatorname{Im} R_{+}+\operatorname{Im} K\right)}{\exp \left(-2 \operatorname{Im} R_{-}+\operatorname{Im} K\right)} \\
& =\exp \left(\frac{-4 \pi\left(\omega-\omega_{0}\right)}{f^{\prime}\left(r_{+}\right)}\right)
\end{aligned}
$$

where Im represents the imaginary part of the function, and the Hawking temperature is

$$
T_{H}=\frac{f^{\prime}\left(r_{+}\right)}{4 \pi} .
$$

However, above calculation is worked on semiclassical approximation, because we ignored all higher order terms of $\mathcal{O}(\hbar)$. Recently, Banerjee and Majhi proposed a new method beyond semiclassical approximation to research the quantum tunneling, and their works show that the conclusion should be corrected [56-65], and this correct entropy may be applied in quantum gravity theory. Now let us generalize this work in higher-dimensional Reissner-Nordström black hole spacetime.

Because the tetrad $e_{\mu}{ }^{a}$ in the space-time are given by

$$
e_{\mu}{ }^{a}=\operatorname{diag}\left(\sqrt{f}, \frac{1}{\sqrt{f}}, r, r \sin \theta_{1}, \ldots, r \prod_{i=1}^{n-1} \sin \theta_{i}\right),
$$

so that $\Gamma_{\mu}$ is

$$
\begin{aligned}
\widetilde{\gamma}^{a} e_{a}{ }^{\mu} \Gamma_{\mu}= & \widetilde{\gamma}^{1} \sqrt{f}\left(\frac{n}{2 r}+\frac{f^{\prime}}{4 f}\right) \\
& +\frac{1}{2 r} \sum_{k=1}^{n-1} \tilde{\gamma}^{k+1} \frac{(n-k) \cot \theta_{k}}{\prod_{i=1}^{k-1} \sin \theta_{i}} .
\end{aligned}
$$

It means the Dirac equation becomes

$$
\begin{aligned}
& i \frac{\tilde{\gamma}^{0}}{\sqrt{f}}\left(\frac{\partial}{\partial t}+\frac{i q A_{t}}{\hbar}\right) \Psi+\tilde{\gamma}^{1} \sqrt{f}\left(\frac{\partial}{\partial r}+\frac{n}{2 r}+\frac{f^{\prime}}{4 f}\right) \Psi \\
& +\sum_{k=1}^{n-1} \frac{\widetilde{\gamma}^{k+1}}{r \prod_{i=1}^{k-1} \sin \theta_{i}}\left(\frac{\partial}{\partial \theta_{k}}+\frac{(n-k) \cot \theta_{k}}{2}\right) \Psi \\
& +\frac{\widetilde{\gamma}^{n+1}}{r \prod_{i=1}^{n-1} \sin \theta_{i}} \frac{\partial \Psi}{\partial \theta_{n}}+\frac{m}{\hbar} \Psi=0,
\end{aligned}
$$

and this equation can be simplified at event horizon

$$
i \widetilde{\gamma}^{0}\left(\frac{\partial}{\partial t}+\frac{i q A_{0}}{\hbar}\right) \Psi+\widetilde{\gamma}^{1}\left(\frac{\partial}{\partial r_{*}}+\frac{f^{\prime}}{4}\right) \Psi=0,
$$

because $f \rightarrow 0$ at event horizon, and $d r_{*}=d r / f$ is tortoise coordinate. On the other hand, the space-time background is static, and $\Psi$ can be rewritten as

$$
\Psi=\left[\begin{array}{c}
A(r) \\
B(r)
\end{array}\right] e^{-(i / \hbar) \omega t},
$$

where $A(r)$ and $B(r)$ are matrices with $m / 2 \times 1$ and $\omega$ is frequency or energy of Dirac particle. Finally, applying the definitions of $\widetilde{\gamma}^{0}$ and $\tilde{\gamma}^{1}$, we get

$$
\left(\begin{array}{cc}
\omega-\omega_{0} & \hbar f\left(\frac{\partial}{\partial r}-\frac{f^{\prime}}{4 f}\right) \\
\hbar f\left(\frac{\partial}{\partial r}-\frac{f^{\prime}}{4 f}\right) & -\left(\omega-\omega_{0}\right)
\end{array}\right)\left(\begin{array}{c}
A_{q} \\
B_{q}
\end{array}\right)=0
$$

here $A_{q}$ and $B_{q}$ are $q$ th elements of matrices $A(r)$ and $B(r)$, respectively. Above equation becomes

$$
\frac{\partial B_{q} / \partial r}{\partial A_{q} / \partial r}=\frac{\left(\omega-\omega_{0}\right) A_{q}-\hbar\left(f^{\prime} B_{q} / 4\right)}{-\left(\omega-\omega_{0}\right) B_{q}-\hbar\left(f^{\prime} A_{q} / 4\right)},
$$


so

$$
\begin{aligned}
& \frac{\omega-\omega_{0}}{2} \frac{\partial}{\partial r}\left(A_{q}^{2}+B_{q}^{2}\right)-\hbar \frac{f^{\prime}}{4}\left(B_{q} \frac{\partial A_{q}}{\partial r}-A_{q} \frac{\partial B_{q}}{\partial r}\right) \\
& =0 .
\end{aligned}
$$

At event horizon $f^{\prime}\left(r_{0}\right) \neq 0$ and depends on the position $r_{0}$, so above equation implies

$$
\begin{aligned}
\frac{\partial}{\partial r}\left(A_{q}^{2}+B_{q}^{2}\right) & =0, \\
B_{q} \frac{\partial A_{q}}{\partial r}-A_{q} \frac{\partial B_{q}}{\partial r} & =0,
\end{aligned}
$$

and the solution is

$$
A_{q}^{2}+B_{q}^{2}=0
$$

Above relation means $A_{q}$ and $B_{q}$ can be rewritten as

$$
\begin{gathered}
A_{q}=C_{q} e^{(i / \hbar) R_{q}(r)}, \\
B_{q}=F_{q} e^{(i / \hbar) R_{q}(r)},
\end{gathered}
$$

and $C_{q}= \pm i F_{q}$ are constants.

Next, let us use the method beyond semiclassical approximation to expand $R_{q}(r)$ and $K=\omega-\omega_{0}$ as

$$
\begin{aligned}
& R_{q}=R_{q 0}+\sum_{i=1}^{\infty} \hbar^{i} R_{q i}(r), \\
& K=\omega-\omega_{0}=K_{0}+\sum_{i=1}^{\infty} \hbar^{i} K_{i},
\end{aligned}
$$

so we get

$$
\begin{aligned}
& \hbar^{0}:\left(\begin{array}{cc}
-i \frac{K_{0}}{f} & \frac{\partial R_{q 0}}{\partial r} \\
\frac{\partial R_{q 0}}{\partial r} & -i \frac{K_{0}}{f}
\end{array}\right)\left(\begin{array}{c}
C_{q} \\
F_{q}
\end{array}\right)=0, \\
& \hbar^{1}:\left(\begin{array}{cc}
-i \frac{K_{1}}{f} & \frac{\partial R_{q 1}}{\partial r}+\frac{i f^{\prime}}{4 f} \\
\frac{\partial R_{q 1}}{\partial r}+\frac{i f^{\prime}}{4 f} & -i \frac{K_{1}}{f}
\end{array}\right)\left(\begin{array}{c}
C_{q} \\
F_{q}
\end{array}\right)=0, \\
& \hbar^{k}:\left(\begin{array}{cc}
-i \frac{K_{k}}{f} & \frac{\partial R_{q k}}{\partial r} \\
\frac{\partial R_{q k}}{\partial r} & -i \frac{K_{k}}{f}
\end{array}\right)\left(\begin{array}{c}
C_{q} \\
F_{q}
\end{array}\right)=0,
\end{aligned}
$$

Therefore, the determinants of matrices vanish:

$$
\begin{aligned}
\hbar^{0}: R_{q 0 \pm} & = \pm \int \frac{K_{0}}{f} d r \\
\hbar^{1}: R_{q 1 \pm} & = \pm \int \frac{K_{1}-i f^{\prime}\left(r_{+}\right) / 4}{f} d r \\
\hbar^{k}: R_{q k \pm} & = \pm \int \frac{K_{k}}{f} d r, \quad k \geq 2, \\
\operatorname{Im} R_{q i} & =\operatorname{Im} R_{q i+}-\operatorname{Im} R_{q i-}=\frac{2 \pi K_{i}}{f^{\prime}\left(r_{+}\right)} .
\end{aligned}
$$

In order to calculate the tunneling rate and Hawking temperature, we rewrite $\operatorname{Im} R_{q i}=\left(\beta_{i} / A_{h}^{i}\right) \operatorname{Im} R_{q 0}\left(i \geq 0, A_{h}\right.$ is area of black hole, and $\beta_{i}$ are dimensionless constant parameters) since the forms of $R_{q j}$ are the same, so the total radial action is

$$
\begin{aligned}
\operatorname{Im} R_{q} & =\operatorname{Im} R_{q 0}(r)+\sum_{i=1}^{\infty} \hbar^{i} R_{q i}(r) \\
& =\left(1+\sum_{i=1}^{\infty} \beta_{i} \frac{\hbar^{i}}{A_{h}^{i}}\right) R_{q 0} .
\end{aligned}
$$

The tunneling rate of Dirac particle at event horizon is given by

$$
\begin{aligned}
\bar{\Gamma}_{h} & =\exp \left[-\frac{2}{\hbar}\left(1+\sum_{i=1}^{\infty} \beta_{i} \frac{\hbar^{i}}{A_{h}^{i}}\right) R_{q 0}\right] \\
& =\exp \left[-\frac{4 \pi K_{0}}{\hbar f^{\prime}\left(r_{+}\right)}\left(1+\sum_{i=1}^{\infty} \beta_{i} \frac{\hbar^{i}}{A_{h}^{i}}\right)\right] .
\end{aligned}
$$

From the relation between tunneling rate and Hawking radiation, we get the temperature of black holes

$$
T_{h}=\left(1+\sum_{i=1}^{\infty} \beta_{i} \frac{\hbar^{i}}{A_{h}^{i}}\right) T_{H} .
$$

Finally, the laws of black hole thermodynamics request

$$
\begin{aligned}
S_{h} & =\int d S_{h}=\left.\int \frac{d M-A d Q}{T_{h}}\right|_{r=r_{+}} \\
& =\frac{A_{h}}{4 \pi}+\pi \beta_{1} \ln \left(A_{h}\right)+\cdots \\
& =S_{H}+\pi \beta_{1} \ln \left(S_{H}\right)+\cdots
\end{aligned}
$$

and $S_{H}=A_{h} / 4 \pi$ is entropy of semiclassical approximation, and this result shows the correction of entropy is logarithmic correction.

In this paper, we studied fermions tunneling from higherdimensional Reissner-Nordström black holes and obtained the Hamilton-Jacobi equation from charged Dirac equation. This work shows that semiclassical Hamilton-Jacobi equation can describe the property of both 0 spin scalar particles 
and $1 / 2$ spin fermions. In this work, we did not emphasize dimensions of space-time larger than $(3+1)$ dimensions, so the method also can be used in the research of $(3+1)$ dimensions and lower cases.

As we all know, the information loss is an open problem in black hole physics, and the information of particles maybe vanishes at the singularity. In order to solve this difficulty, Horowitz and Maldacena proposed a boundary condition, which is called the black hole final state, at singularity of black hole to perfectly entangle the incoming Hawking radiation particles and the collapsing matter $[66,67]$. Due to the boundary condition, any particle which is falling into the black holes completely annihilates. It is a new and interesting idea to investigate the black hole physics and quantum gravity, so we also will work on this area in the future.

\section{Competing Interests}

The authors declare that they have no competing interests.

\section{Acknowledgments}

This work is supported in part by FAPESP no. 2012/08934-0, CNPq, CAPES, and National Natural Science Foundation of China (no. 11573022 and no. 11375279).

\section{References}

[1] S. W. Hawking, "Black hole explosions?" Nature, vol. 248, no. 5443, pp. 30-31, 1974.

[2] S. W. Hawking, "Particle creation by black holes," Communications in Mathematical Physics, vol. 43, no. 3, pp. 199-220, 1975.

[3] S. P. Robinson and F. Wilczek, "Relationship between Hawking radiation and gravitational anomalies," Physical Review Letters, vol. 95, no. 1, 011303, 4 pages, 2005.

[4] T. Damoar and R. Ruffini, "Black-hole evaporation in the KleinSauter-Heisenberg-Euler formalism," Physical Review D, vol. 14, no. 2, p. 332, 1976.

[5] S. Sannan, "Heuristic derivation of the probability distributions of particles emitted by a black hole," General Relativity and Gravitation, vol. 20, no. 3, pp. 239-246, 1988.

[6] P. Kraus and F. Wilczek, "Self-interaction correction to black hole radiance," Nuclear Physics B, vol. 433, no. 2, pp. 403-420, 1995.

[7] M. K. Parikh and F. Wilczek, "Hawking radiation as tunneling," Physical Review Letters, vol. 85, no. 24, pp. 5042-5045, 2000.

[8] S. Hemming and E. Keski-Vakkuri, "Hawking radiation from AdS black holes," Physical Review D, vol. 64, no. 4, Article ID 044006, 8 pages, 2001.

[9] Q.-Q. Jiang, S.-Q. Wu, and X. Cai, "Hawking radiation from dilatonic black holes via anomalies," Physical Review D, vol. 75, no. 6, Article ID 064029, 10 pages, 2007.

[10] S.-Q. Wu and Q.-Q. Jiang, "Hawking radiation of charged particles as tunneling from higher dimensional ReissnerNordstrom-de Sitter black holes," https://arxiv.org/abs/hep-th/ 0603082.

[11] R. Kerner and R. B. Mann, "Tunnelling, temperature, and TaubNUT black holes," Physical Review D, vol. 73, no. 10, Article ID 104010, 2006.
[12] J. Ren, J. Y. Zhang, and Z. Zhao, "Tunnelling effect and hawking radiation from a Vaidya black hole," Chinese Physics Letters, vol. 23, no. 8, pp. 2019-2022, 2006.

[13] S. Iso, H. Umetsu, and F. Wilczek, "Anomalies, Hawking radiations, and regularity in rotating black holes," Physical Review D, vol. 74, no. 4, Article ID 044017, 10 pages, 2006.

[14] S. Iso, T. Morita, and H. Umetsu, "Quantum anomalies at horizon and Hawking radiations in Myers-Perry black holes," Journal of High Energy Physics, vol. 2007, no. 4, article 068, 14 pages, 2007.

[15] S. Z. Yang, H. L. Li, Q. Q. Jiang, and M. Q. Liu, “The research on the quantum tunneling characteristics and the radiation spectrum of the stationary axisymmetric black hole," Science in China Series G, vol. 50, no. 2, pp. 249-260, 2007.

[16] E. C. Vagenas, "Are extremal 2D black holes really frozen?" Physics Letters B, vol. 503, no. 3-4, pp. 399-403, 2001.

[17] E. C. Vagenas, "Two-dimensional dilatonic black holes and Hawking radiation," Modern Physics Letters A, vol. 17, no. 10, pp. 609-618, 2002.

[18] E. C. Vagenas, "Semiclassical corrections to the BekensteinHawking entropy of the BTZ black hole via self-gravitation," Physics Letters B, vol. 533, no. 3-4, pp. 302-306, 2002.

[19] A. J. M. Medved, "Radiation via tunnelling in the charged BTZ black hole," Classical and Quantum Gravity, vol. 19, no. 3, pp. 589-598, 2002.

[20] M. K. Parikh, "New coordinates for de Sitter space and de Sitter radiation," Physics Letters B, vol. 546, no. 3-4, pp. 189-195, 2002.

[21] A. J. M. Medved, "Radiation via tunneling from a de Sitter cosmological horizon," Physical Review D, vol. 66, no. 12, Article ID 124009, 2002.

[22] E. C. Vagenas, "Generalization of the KKW analysis for black hole radiation," Physics Letters B, vol. 559, no. 1-2, pp. 65-73, 2003.

[23] M. K. Parikh, "Energy conservation and Hawking radiation," https://arxiv.org/abs/hep-th/0402166.

[24] J. Y. Zhang and Z. Zhao, "Massive particles' Hawking radiation via tunneling," Acta Physica Sinica, vol. 55, p. 3796, 2006 (Chinese).

[25] J. Y. Zhang and Z. Zhao, "Charged particles' tunnelling from the Kerr-Newman black hole," Physics Letters B, vol. 638, no. 2-3, pp. 110-113, 2006.

[26] E. T. Akhmedov, V. Akhmedova, D. Singleton, and T. Pilling, "Thermal radiation of various gravitational backgrounds," International Journal of Modern Physics A, vol. 22, no. 8-9, pp. 1705$1715,2007$.

[27] E. T. Akhmedov, V. Akhmedova, and D. Singleton, "Hawking temperature in the tunneling picture," Physics Letters B, vol. 642, no. 1-2, pp. 124-128, 2006.

[28] B. D. Chowdhury, "Problems with tunneling of thin shells from black holes," Pramana, vol. 70, no. 1, pp. 3-26, 2008.

[29] E. T. Akhmedov, T. Pilling, and D. Singleton, "Subtleties in the quasi-classical calculation of hawking radiation," International Journal of Modern Physics D, vol. 17, no. 13-14, p. 2453, 2008.

[30] V. Akhmedova, T. Pilling, A. de Gill, and D. Singleton, "Temporal contribution to gravitational WKB-like calculations," Physics Letters B, vol. 666, no. 3, pp. 269-271, 2008.

[31] V. Akhmedova, T. Pilling, A. de Gill, and D. Singleton, "Comments on anomaly versus WKB/tunneling methods for calculating Unruh radiation,” Physics Letters B, vol. 673, no. 3, pp. 227-231, 2009. 
[32] K. Srinivasan and T. Padmanabhan, "Particle production and complex path analysis," Physical Review D, vol. 60, Article ID 024007, 1999.

[33] S. Shankaranarayanan, T. Padmanabhan, and K. Srinivasan, "Hawking radiation in different coordinate settings: complex paths approach," Classical and Quantum Gravity, vol. 19, no. 10, pp. 2671-2687, 2002.

[34] M. Angheben, M. Nadalini, L. Vanzo, and S. Zerbini, "Hawking radiation as tunneling for extremal and rotating black holes," Journal of High Energy Physics (JHEP), vol. 505, p. 14, 2005.

[35] S. Z. Yang and D. Y. Chen, "Hawking radiation as tunneling from the Vaidya-Bonner black hole," International Journal of Theoretical Physics, vol. 46, no. 11, pp. 2923-2927, 2007.

[36] D. Y. Chen and S. Z. Yang, "Hawking radiation of the VaidyaBonner-de Sitter black hole," New Journal of Physics, vol. 9, no. 8, p. 252, 2007.

[37] R. Kerner and R. B. Mann, "Fermions tunnelling from black holes," Classical and Quantum Gravity, vol. 25, no. 9, 095014, 17 pages, 2008.

[38] R. Kerner and R. B. Mann, "Charged fermions tunnelling from Kerr-Newman black holes," Physics Letters B, vol. 665, no. 4, pp. 277-283, 2008.

[39] R. Li, J. R. Ren, and S. W. Wei, "Hawking radiation of Dirac particles via tunneling from the Kerr black hole," Classical and Quantum Gravity, vol. 25, no. 12, Article ID 125016, 1250.

[40] R. Li and J.-R. Ren, "Dirac particles tunneling from BTZ black hole," Physics Letters B, vol. 661, no. 5, pp. 370-372, 2008.

[41] D.-Y. Chen, Q.-Q. Jiang, and X.-T. Zu, "Fermions tunnelling from the charged dilatonic black holes," Classical and Quantum Gravity, vol. 25, no. 20, Article ID 205022, 2008.

[42] D.-Y. Chen, Q.-Q. Jiang, and X.-T. Zu, "Hawking radiation of Dirac particles via tunnelling from rotating black holes in de Sitter spaces," Physics Letters B, vol. 665, no. 2-3, pp. 106-110, 2008.

[43] X.-X. Zeng and S.-Z. Yang, "Fermions tunneling from ReissnerNordström black hole," General Relativity and Gravitation, vol. 40, no. 10, pp. 2107-2114, 2008.

[44] K. Lin and S. Z. Yang, "Fermions tunneling of the VaidyaBonner black hole," Acta Physica Sinica, vol. 58, no. 2, pp. 744748, 2009 (Chinese).

[45] R. Di Criscienzo and L. Vanzo, "Fermion tunneling from dynamical horizons," Europhysics Letters, vol. 82, no. 6, Article ID 60001, 2008.

[46] L. H. Li, S. Z. Yang, T. J. Zhou, and R. Lin, "Fermion tunneling from a Vaidya black hole," EPL (Europhysics Letters), vol. 84, no. 2, p. 20003, 2008.

[47] Q.-Q. Jiang, "Fermions tunnelling from GHS and non-extremal D1-D5 black holes," Physics Letters B, vol. 666, no. 5, pp. 517-521, 2008.

[48] Q.-Q. Jiang, "Dirac particle tunneling from black rings," Physical Review D, vol. 78, no. 4, Article ID 044009, 8 pages, 2008.

[49] K. Lin and S. Z. Yang, "Fermion tunneling from higherdimensional black holes," Physical Review D, vol. 79, no. 6, Article ID 064035, 2009.

[50] K. Lin and S. Z. Yang, "Fermions tunneling of higherdimensional Kerr-anti-de Sitter black hole with one rotational parameter," Physics Letters B, vol. 674, no. 2, pp. 127-130, 2009.

[51] F. R. Tangherlini, "Schwarzschild field inn dimensions and the dimensionality of space problem," Il Nuovo Cimento, vol. 27, no. 3, pp. 636-651, 1963.
[52] S. J. Gao and J. P. Lemos, "Collapsing and static thin massive charged dust shells in a Reissner-Nordström black hole background in higher dimensions," International Journal of Modern Physics A, vol. 23, no. 19, pp. 2943-2960, 2008.

[53] R. A. Konoplya and A. Zhidenko, "Stability of multidimensional black holes: complete numerical analysis," Nuclear Physics B, vol. 777, no. 1-2, pp. 182-202, 2007.

[54] R. A. Konoplya and A. Zhidenko, "Instability of higherdimensional charged black holes in the de Sitter world," Physical Review Letters, vol. 103, no. 16, Article ID 161101, 4 pages, 2009.

[55] R. A. Konoplya and A. Zhidenko, "Stability of higher dimensional Reissner-Nordström-anti-de Sitter black holes," Physical Review D, vol. 78, no. 10, Article ID 104017, 9 pages, 2008.

[56] R. Banerjee and B. R. Majhi, "Quantum tunneling beyond semiclassical approximation," Journal of High Energy Physics, vol. 2008, no. 6, article 095, 2008.

[57] R. Banerijee and B. R. Majhi, "Quantum tunneling and trace anomaly," Physics Letters B, vol. 674, no. 3, pp. 218-222, 2009.

[58] B. R. Majhi, "Fermion tunneling beyond semiclassical approximation," Physical Review D, vol. 79, no. 4, Article ID 044005, 2009.

[59] R. Banerjee and B. R. Majhi, "Connecting anomaly and tunneling methods for the Hawking effect through chirality," Physical Review D, vol. 79, no. 6, Article ID 064024, 5 pages, 2009.

[60] R. Banerijee, B. R. Majhi, and D. Roy, "Corrections to Unruh effect in tunneling formalism and mapping with Hawking effect," https://arxiv.org/abs/0901.0466.

[61] B. R. Majhi and S. Samanta, "Hawking radiation due to photon and gravitino tunneling," Annals of Physics, vol. 325, no. 11, pp. 2410-2424, 2010.

[62] R. Banerijee and B. R. Majhi, "Hawking black body spectrum from tunneling mechanism," Physics Letters B, vol. 675, no. 2, pp. 243-245, 2009.

[63] R. Banerjee and B. R. Majhi, "Quantum tunneling and back reaction," Physics Letters B, vol. 662, no. 1, pp. 62-65, 2008.

[64] R. Banerjee, B. R. Majhi, and S. Samanta, "Noncommutative black hole thermodynamics," Physical Review D, vol. 77, no. 12, Article ID 124035, 8 pages, 2008.

[65] S. K. Modak, "Corrected entropy of BTZ black hole in tunneling approach," Physics Letters B, vol. 671, no. 1, pp. 167-173, 2009.

[66] G. T. Horowitz and J. Maldacena, "The black hole final state," Journal of High Energy Physics, vol. 2004, no. 2, article 008, 15 pages, 2004.

[67] G. T. Horowitz and E. Silverstein, “The inside story: quasilocal tachyons and black holes," Physical Review D, vol. 73, no. 6, Article ID 064016, 14 pages, 2006. 

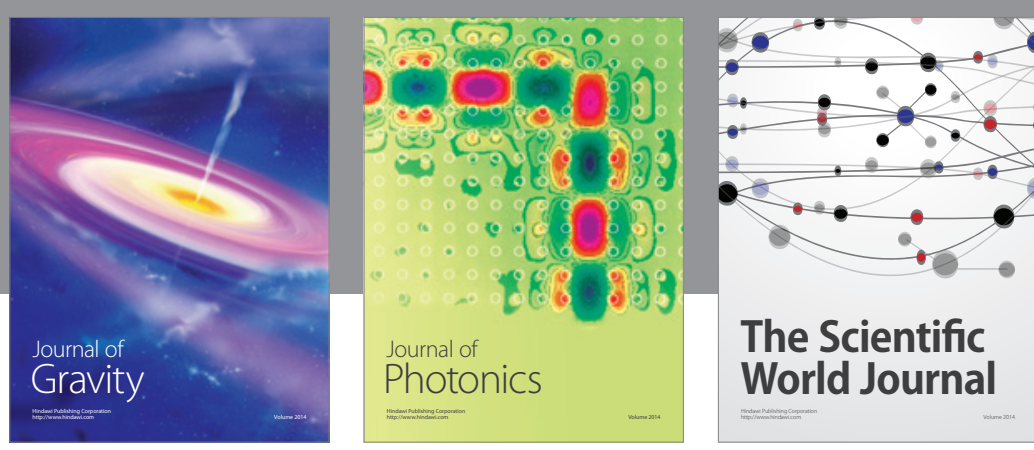

The Scientific World Journal
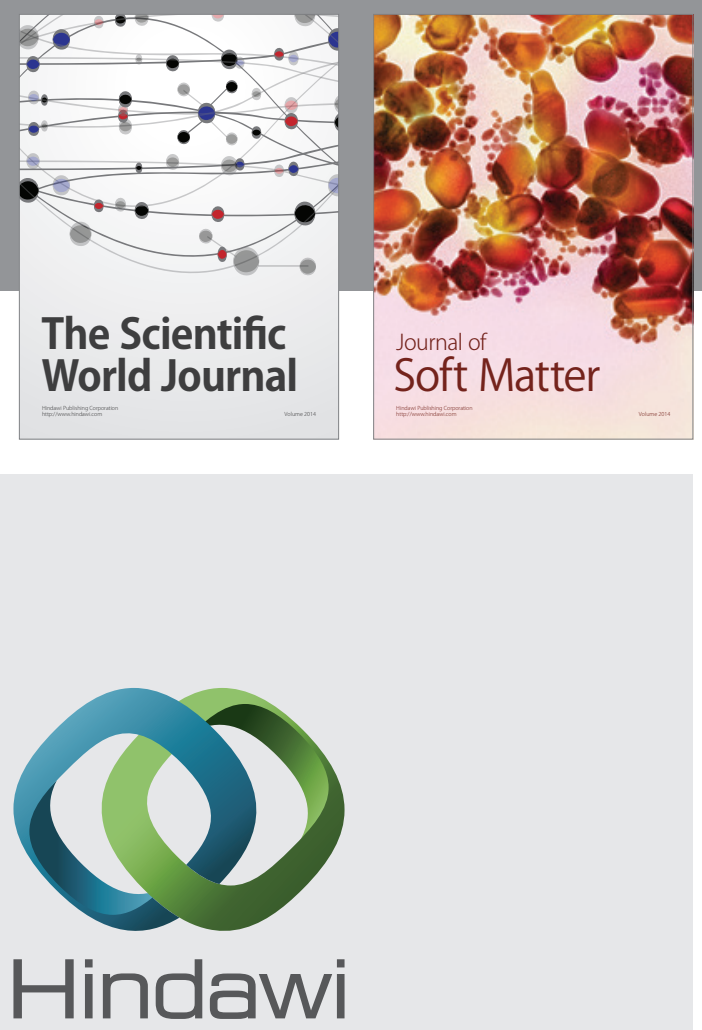

Submit your manuscripts at

http://www.hindawi.com

nternational Journal of

Statistical Mechanics
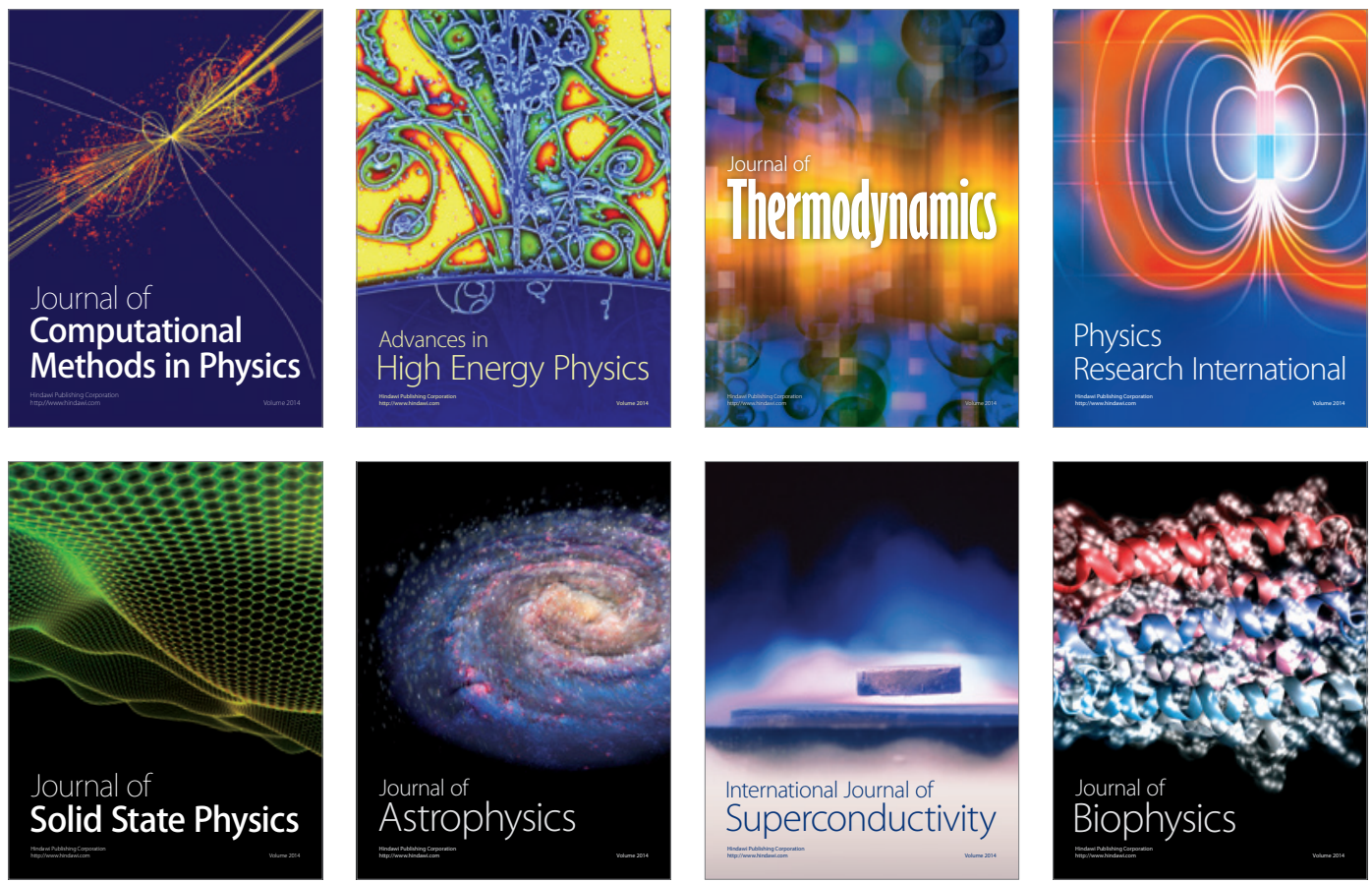
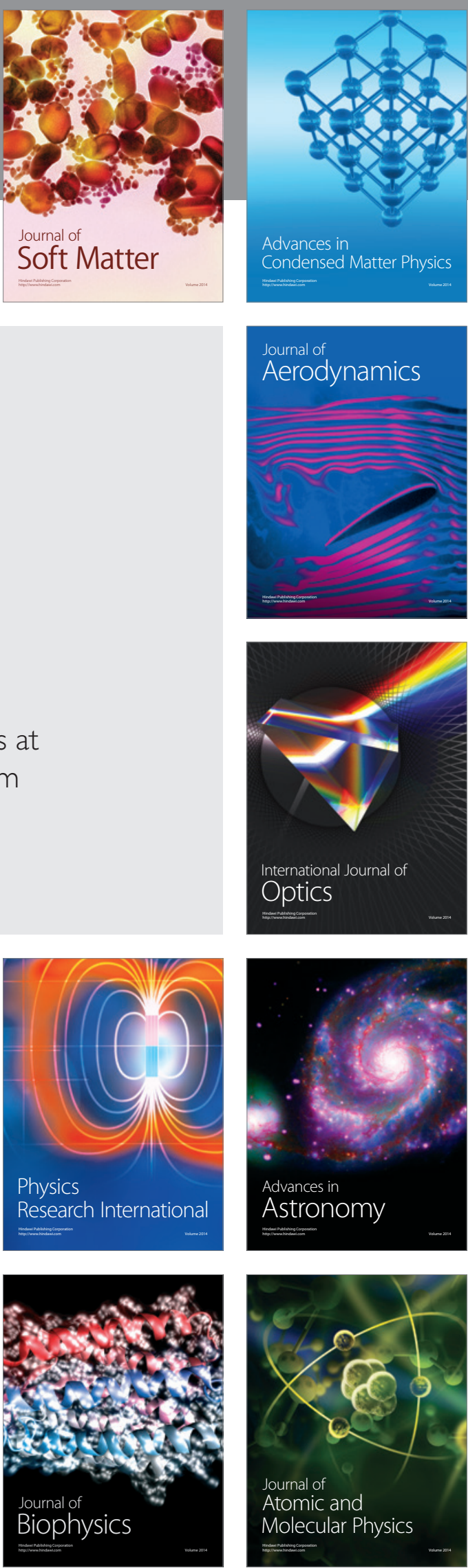